\title{
Complementary Nucleobase Interactions Drive the Hierarchical Self-Assembly of Core- Shell Bottlebrush Block Copolymers Toward Cylindrical Supramolecules
}

\author{
Spyridon Varlas, ${ }^{\dagger, \nabla}$ Zan Hua, ${ }^{\star}, \nabla$ Joseph R. Jones, ${ }^{\dagger}$ Marjolaine Thomas, ${ }^{\dagger}$ Jeffrey C. Foster, ${ }^{* \dagger}{ }^{\dagger}$ and Rachel K. \\ O’Reilly*,† \\ †School of Chemistry, University of Birmingham, Edgbaston, Birmingham, B15 2TT, UK \\ Department of Chemistry, University of Warwick, Gibbet Hill Road, Coventry, CV4 7AL, UK
}

\begin{abstract}
The self-assembly of amphiphilic block copolymers has facilitated the preparation of a wide variety of nano-objects of diverse morphology. Ready access to these nanostructures has opened up new possibilities in catalysis, sensing and nanomedicine. In comparison, the self-assembly of large building blocks (i.e., amphiphilic bottlebrush polymers) has received less attention, owing in part to the relatively more challenging synthesis of these macromolecules. Bottlebrush amphiphiles can self-assemble into uniquely stable spherical nanostructures and can also produce dynamic cylinders with lengths modulated by environmental conditions, motivating further research in this area. Herein, we report the synthesis of core-shell bottlebrush polymers (BBPs) containing complementary nucleobase functionalities via a combination of ring-opening metathesis polymerization (ROMP) and reversible addition-fragmentation chain transfer (RAFT) polymerization, using a "grafting-from" approach, and their hierarchical selfassembly in aqueous media. Mixtures of BBPs containing thymine or adenine units in their core blocks were found to self-assemble into higher-order cylindrical supramolecules upon heating above a critical temperature. This temperature was demonstrated to correspond to the lower critical solution temperature (LCST) of the corona-forming poly(4-acryloylmorpholine) block, providing evidence for a unique onedimensional BBP assembly mechanism. Moreover, the formation of extended supramolecular assemblies was preferentially observed when both thymine- and adenine-functionalized BBPs were present in equimolar concentrations, pointing towards an alternating, isodesmic mechanism of organization occurring
\end{abstract}


via nucleobase interactions located at their chain termini. We anticipate that these discoveries will provide the basis for future studies regarding BBP self-assembly, especially with regards to the formation of stimuliresponsive anisotropic nanostructures.

\section{INTRODUCTION}

Nature has perfected the creation of sophisticated constructs at the nanometer scale through the hierarchical assembly of biomacromolecules. ${ }^{1-2}$ These self-assembly processes yield a broad variety of structures from one-dimensional tubules and fibers to helices to even more complex three-dimensional structures, such as those possessed by proteins, wherein the precise positioning of key functionalities modulates reactivity in chemical transformations. ${ }^{3}$ In addition to their precise structures, nature's assemblies are dynamic, capable of undergoing reversible changes in their degree of association, conformation, or length in response to physiologically relevant stimuli. ${ }^{4-5}$ For instance, the dynamic assembly of $\alpha$ - and $\beta$-tubulin heterodimers mediates processes in the cytoskeleton such as cell motility, intracellular transport, and cell division. ${ }^{6}$ This non-equilibrium behavior originates from the binding and hydrolysis of the nucleotide guanosine-5'triphosphate (GTP), which enables their polymerization or depolymerization by modulating tubulin subunits at the ends of the microtubules.

Self-assembly procedures of synthetic, nanostructured building blocks into long, anisotropic supramolecules which mimic the behavior of microtubules or fibers have long been of interest to the scientific community. ${ }^{7-8}$ Amphiphilic block copolymers with crystallizable segments, ${ }^{9-11}$ peptide amphiphiles, ${ }^{12-13}$ cyclic peptides, ${ }^{14-16}$ macrocycles, ${ }^{17-19}$ and dendrons ${ }^{20-21}$ have all been exploited for the preparation of synthetic microtubes and fibers. Indeed, a broad range of supramolecular nanostructures have been accessed using these building blocks, with excellent control over dimension, topology, and surface/internal functionality. ${ }^{22-24}$ However, the factors underlying control over the supramolecular assembly of relatively larger building blocks (i.e., the secondary assembly of semi-crystalline cylindrical micelles, peptide nanofibers, or bottlebrush polymers) are, in comparison, less well-understood. ${ }^{25-26}$ 
The study of bottlebrush polymer (BBP) self-assembly mechanisms has accelerated in recent years, driven by the architectural control afforded by controlled polymerization techniques. ${ }^{27}$ BBPs are large, single macromolecules comprised of numerous polymer side-chains grafted to a polymer backbone. ${ }^{7,28}$ The often dense grafting of their side-chains along with their persistent cylindrical shape makes them ideal candidates for the preparation of nanostructured materials. To date, the majority of literature reports have focused on the self-assembly of amphiphilic bottlebrush di- or triblock copolymers (BBCPs), which behave analogously to linear block copolymers with the exception of scale. ${ }^{29-30}$ For instance, giant surfactants prepared from amphiphilic BBCPs were shown to self-assemble into spherical micelles, ${ }^{31}$ cylindrical microdomains with remarkably long range order could be prepared from Janus-type BBCPs,${ }^{32}$ and photonic crystals were found to arise during bulk self-assembly of BBCPs within thin films due to the large dimensions of the phase-separated domains (on the order of the wavelength of visible light). ${ }^{33-34}$ In each of these examples, the self-assembly behavior is governed by the immiscibility of polymer side-chains within discrete blocks.

In contrast, amphiphilic core-shell BBPs, which possess an internal solvophobic compartment near the BBP backbone and an outer solvophilic layer, have been demonstrated to self-assemble in an end-to-end fashion. ${ }^{35-36}$ In this case, non-covalent intermolecular interactions between solvophobic regions at each chain-end drive the formation of higher-order one-dimensional cylindrical structures. This end-to-end selfassembly has been speculated to occur due to the incomplete shielding of the terminal interfaces of the BBPs by the corona chains, imparting a unique anisotropy to BBPs that differentiates their behavior from linear diblock copolymers. End-to-end assembly has been observed for core-shell BBPs with charged coronas, driven by hydrophobic interactions between polystyrene cores (Figure 1A), ${ }^{35}$ and for BBPs with carboxyl functionalities in their core and coronae comprised of polyethylene glycol units that self-assemble through intermolecular H-bonding under acidic conditions. ${ }^{36}$ In both cases, spontaneous self-assembly was observed without application of an external stimulus. 
Based on these initial reports, we wondered whether selectivity could be imparted on the end-to-end assembly of core-shell BBPs by incorporating nucleobase functionality. Nucleobases are the components of DNA and RNA that confer specific and complementary interactions between strands. For instance, thymine (T) and adenine (A) form a purine-pyrimidine pair known as a base complement in which multiple hydrogen bonds effect selective cross-pairing. Nucleobase functionalization of polymers has been utilized extensively in the preparation of supramolecular materials and nanoparticles. ${ }^{37-40}$ Previous reports from our group have demonstrated the preservation of selective nucleobase interactions between amphiphilic block copolymers containing acrylamide blocks fitted with either thymine or adenine, which, when mixed, exhibited unique self-assembly behavior. ${ }^{41-45}$ In this context, we envisioned that amphiphilic core-shell BBPs equipped with nucleobase functionality within their core compartment could self-assemble in an endto-end fashion, with interfacial interactions between BBPs in the assemblies dominated by complementary nucleobase pairing (Figure 1B). These interactions were hypothesized to confer additional organization to the BBP assemblies, as the BBPs should interact preferentially with those containing complementary nucleobases. 
A. Previous work - assembly driven by hydrophobicity (Wooley et al.)

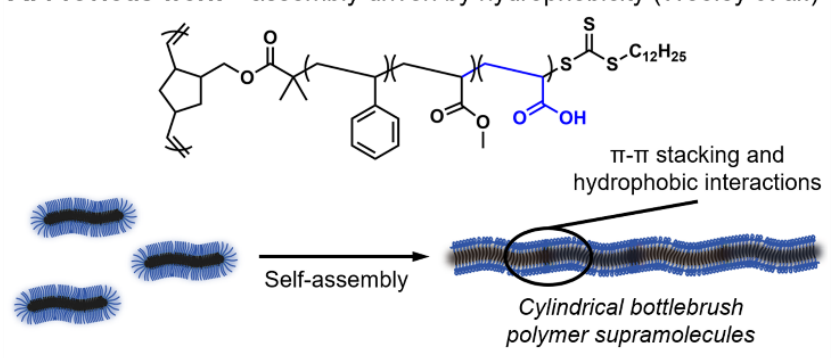

B. This work - assembly driven by hydrophobicity and $\mathrm{H}$-bonding

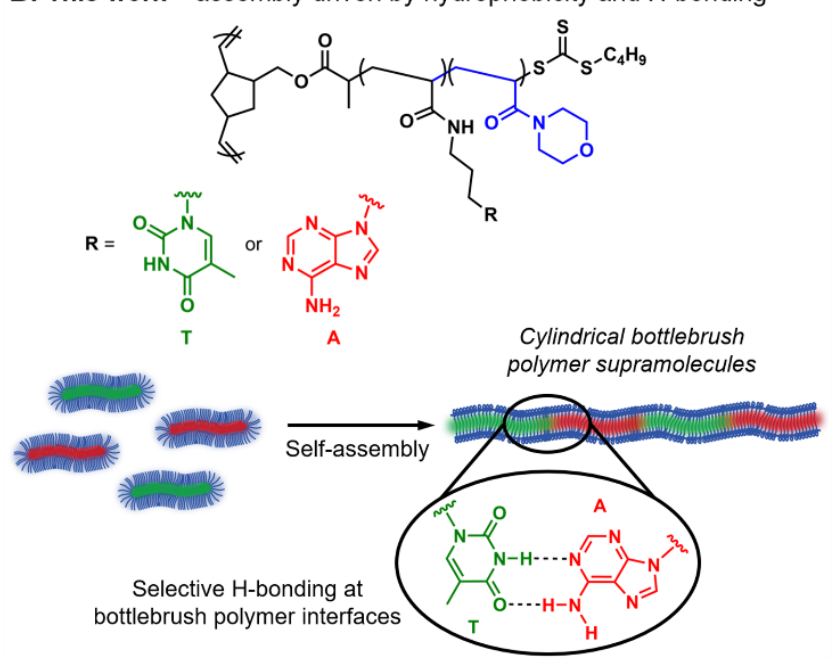

Figure 1. End-to-end supramolecular self-assembly of amphiphilic bottlebrush copolymers (BBCPs) with core-shell architecture. (A) Assembly driven by $\pi$ - $\pi$ stacking and hydrophobic interactions, ${ }^{35}$ and (B) assembly driven by complementary T-A H-bonding and hydrophobic interactions.

Herein, we report the synthesis of amphiphilic thymine- or adenine-containing core-shell BBPs via a combination of ring-opening metathesis polymerization (ROMP) and reversible addition-fragmentation chain-transfer (RAFT) polymerization, using a "grafting-from" procedure. Notably, the nucleobasecontaining BBPs were found to selectively self-assemble to form hierarchical one-dimensional structures when BBPs with complementary nucleobase functionalities were mixed upon heating. In addition, we investigated the extent of selectivity of the assembly process with respect to nucleobase pairing and proposed a general mechanism for the supramolecular end-to-end self-organization of the prepared coreshell BBPs. Finally, the ability of the formed cylindrical supramolecules to retain their characteristics upon 
cross-linking at elevated temperatures and subsequent cooling to room temperature was also demonstrated. The reported mechanism of self-assembly is expected to be general for core-shell BBPs in aqueous solution regardless of the polymer side-chain composition. We anticipate that information regarding the 1D assembly of BBPs will enable the preparation of stimuli-responsive cylindrical nanostructures in future studies and could be of particular interest in the preparation of super-soft, dynamic networked materials.

\section{RESULTS AND DISCUSSION}

To prepare nucleobase-containing core-shell BBPs, we opted to utilize acrylamide-based monomers that bear nucleobase units, in this case either thymine $(\mathrm{T})$ or adenine $(\mathrm{A})$, at the end of a methylene spacer. These monomers have proven useful in previous studies due to their ease of synthesis and well-controlled polymerization. ${ }^{41,43-44}$ The monomers were synthesized in two steps according to our previously reported procedure. ${ }^{42}$ For the BBP corona, 4-acryloylmorpholine (NAM) was chosen. PNAM is produced from a commercially available acrylamide monomer and represents a promising alternative to polyethylene glycol due to its ease of preparation, biocompatibility, potentially interesting pharmacokinetics, and high water solubility in its neutral form. ${ }^{46}$ In addition, previous studies from our group have demonstrated good compatibility of PNAM with nucleobase-functionalized acrylamide polymers. ${ }^{41,43-44}$ Our initial attempts to prepare nucleobase-containing BBPs were conducted using a "grafting-through" polymerization methodology. Macromonomers comprised of either a hydrophobic core-forming poly(thymine acrylamide) (PTAm) or poly(adenine acrylamide) (PAAm) block, a hydrophilic PNAM block, and a norbornene endgroup were prepared via thermally initiated reversible addition-fragmentation chain-transfer (RAFT) polymerization. Consequently, "grafting-through" mediated by ring-opening metathesis polymerization (ROMP) using these macromonomers and $\left(\mathrm{H}_{2} \mathrm{IMes}\right)(\mathrm{Py})_{2}(\mathrm{Cl})_{2} \mathrm{Ru}=\mathrm{CHPh}(\mathbf{G 3})$ was attempted. However, only low conversions (i.e., < 30\%) could be obtained via ROMP "grafting-through" regardless of reaction conditions (various solvents, concentrations, and additives were tested), resulting in BBPs that were 
contaminated with large quantities of unreacted macromonomer that was difficult to remove from the polymer samples.

As an alternative approach, the synthesis of nucleobase-functionalized BBPs was achieved using a "grafting-from" procedure (Figure 2A). First, a poly(chain transfer agent) (PCTA, DP = 100) was prepared via ROMP of a small molecule CTA that had been fitted with a norbornene polymerizable end-group (NBCTA). A block of PTAm or PAAm was then grown via RAFT polymerization using the synthesized PCTA and 2,2'-azobis(2-methylpropionitrile) (AIBN) as a source of radicals to yield either $\mathbf{P N B}_{\mathbf{1 0 0}} \mathbf{g}$-PTAm $\mathbf{1 2}$ or $\mathbf{P N B}_{100}-\boldsymbol{g}$-PAAm 12 BBPs depending on the monomer utilized. Next, chain-extension of these precursor BBPs using NAM via RAFT polymerization under similar reaction conditions yielded amphiphilic PNB $_{\mathbf{1 0 0}^{-}}$ $g$-(PTAm ${ }_{12}-b$-PNAM $\left.{ }_{40}\right)$ and PNB $_{100}-g-\left(\right.$ PAAm $_{12}-b-$ PNAM $\left._{40}\right)$ core-shell BBPs, which contained either T or A nucleobase units, respectively, in their hydrophobic domains. These BPPs will be referred to as TBB (for a PTAm-based core) and ABB (for a PAAm-based core). As shown in Figure 2B, Figure S7, and Table S1, the final BBPs possessed monomodal molecular weight distributions with moderate dispersity values $\left(\bigoplus_{\mathrm{M}, \mathbf{T B B}}=1.44, \bigoplus_{\mathrm{M}, \mathrm{ABB}}=1.60\right)$. High molecular weight shoulders were present in both samples, which could originate from poor solubility of the BBPs in the SEC mobile phase $\left(\mathrm{DMF}+5 \mathrm{mM} \mathrm{NH}_{4} \mathrm{BF}_{4}\right)$ or intermolecular coupling between BBPs that occurred during the RAFT process. In addition, number average molecular weight values $\left(M_{\mathrm{n}}\right)$ determined by SEC analysis were evidently lower than theoretically expected values determined from polymerization conversions. This discrepancy in theoretical and observed $M_{\mathrm{n}}$ likely originates from the highly branched nature of the BBPs, leading to reduced hydrodynamic volumes relative to linear polymers of the same molecular weight and thus increased retention on the SEC columns. ${ }^{1} \mathrm{H}-$ NMR spectra of both TBB and ABB samples in DMSO- $d_{6}$ confirmed the presence of signals that were assigned to protons present in both the inner nucleobase blocks and the outer NAM shell (Figures S4 and S5). The core-shell conformation of the bottlebrush polymers was also evidenced by their solubility in aqueous milieu. Following synthesis, TBB and ABB were purified by preparative SEC to remove linear polymer impurities. 
A.

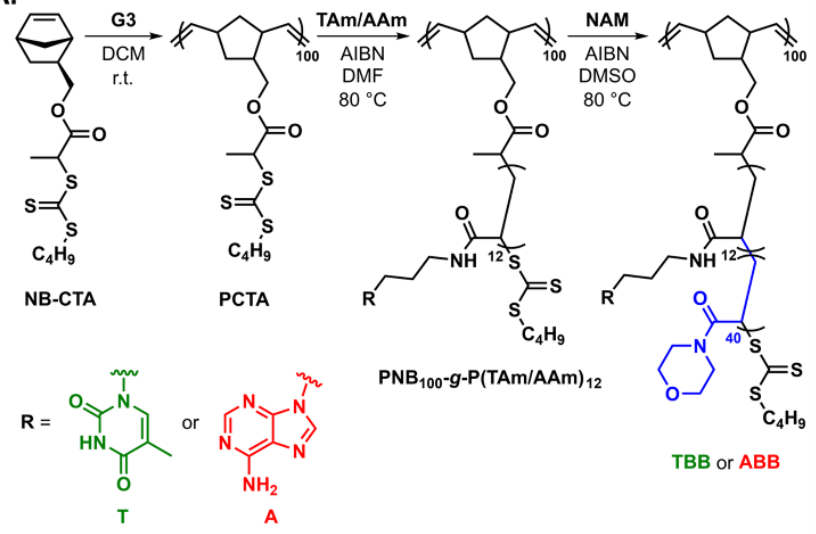

B.

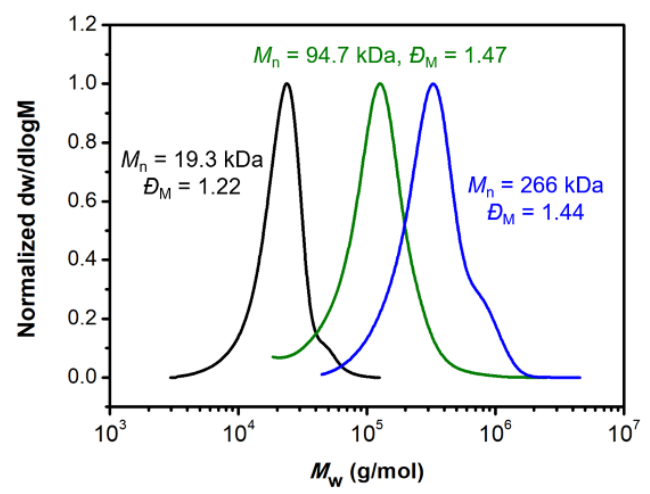

Figure 2. Synthesis of amphiphilic nucleobase-containing core-shell BBPs using a "grafting-from" procedure. (A) Schematic representation of the synthetic route followed for preparation of $\mathbf{T B B}$ and $\mathbf{A B B}$. (B) Normalized SEC RI molecular weight distributions of PCTA (black trace), PNB $_{\mathbf{1 0 0}}$ - $_{\text {-PTAm }}$ (green

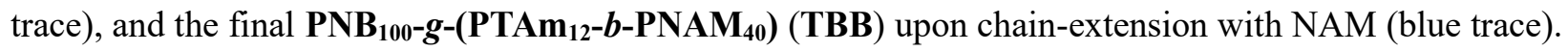
Reported $M_{\mathrm{n}}$ and $\bigoplus_{\mathrm{M}}$ values were calculated from PMMA standards using DMF $+5 \mathrm{mM} \mathrm{NH}_{4} \mathrm{BF}_{4}$ as the eluent.

Importantly, the solution behavior of $\mathbf{T B B}$ and $\mathbf{A B B}$ in aqueous media was further characterized using dynamic light scattering (DLS) and transmission electron microscopy (TEM). As shown in Figures 3A and 3B, both BBP samples exhibited broad size distributions with additional larger components in the DLS traces. The fact that these traces are multi-modal can be rationalized by considering three factors: (1) the presence of coupled bottlebrush polymers which arose during "grafting-from" polymerization (also observed in the SEC traces); (2) a dynamic formation and dissociation of bottlebrush aggregates which 
occurred on the timescale of the DLS experiment; and (3) additional relaxations occurring which contributed to the scattering profile due to the anisotropy of the bottlebrush polymers in solution. ${ }^{47}$ Drystate TEM images of $\mathbf{T B B}$ and $\mathbf{A B B}$ revealed the presence of small spherical nanoparticles, which were primarily single BBP molecules. Particle size analysis was conducted using the acquired images, again revealing broad size distributions for both samples. Sizes measured from TEM were smaller than those obtained from DLS, which is typical for dried samples due to the absence of extended hydration volumes that are present in solution (Table S2). Whilst the BBPs appear to be spherical in the TEM images, they likely exist as cylindrical nanostructures in aqueous environment due to their anisotropic dimensions (the backbone DP is $c a .2 \times$ the length of the combined side-chain DPs), as has been reported for BBPs in solution measured using X-ray or neutron scattering analyses. ${ }^{48-49}$ Moreover, BBPs have been shown to de-wet various substrates due to unfavorable surface interactions, causing them to present as globules in TEM or atomic force microscopy (AFM) images. ${ }^{50}$ Importantly, no large aggregates were observed in the TEM images, indicating that neither BPP spontaneously self-assembled into higher-order structures when dissolved in aqueous media at room temperature. 

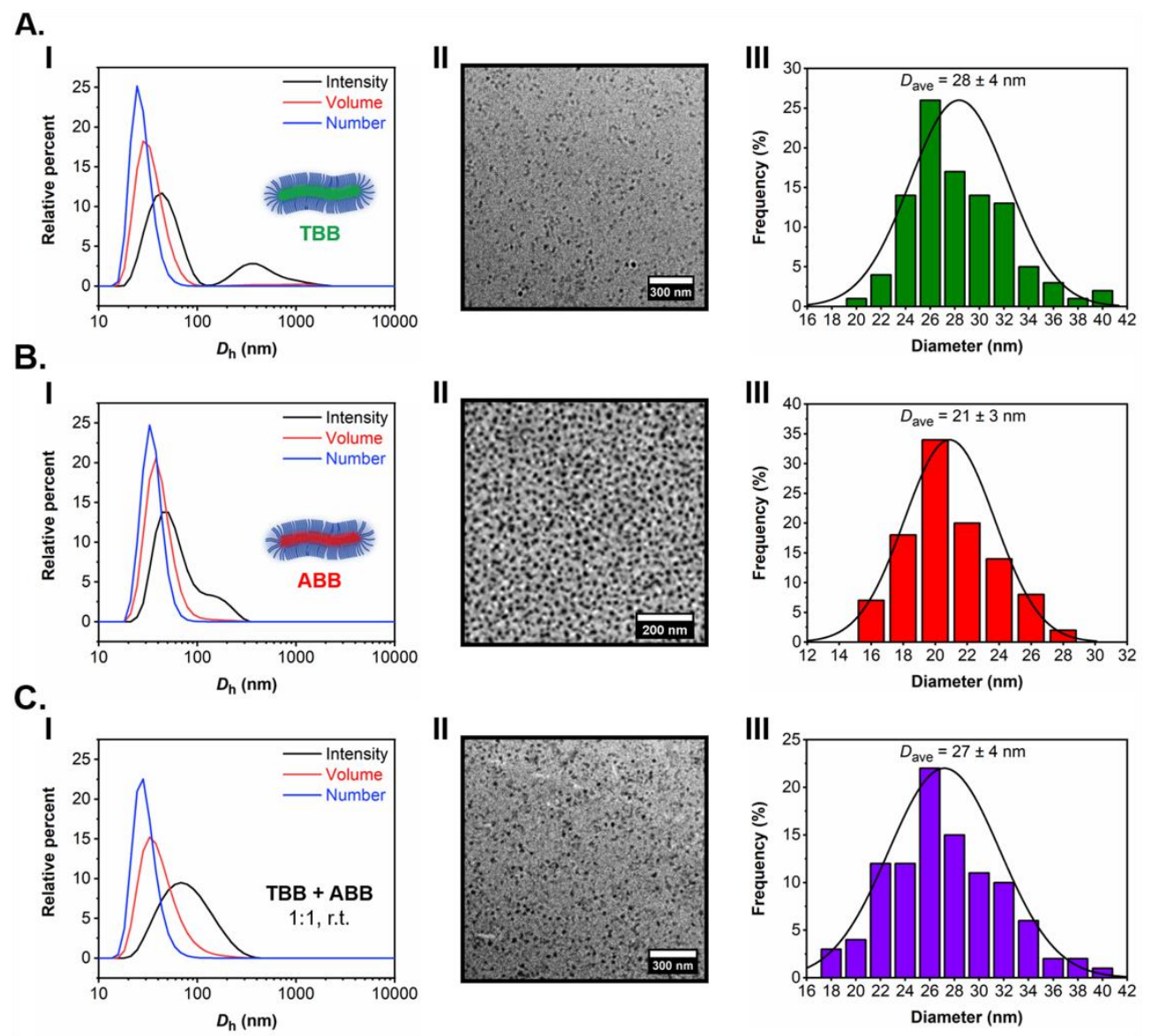

Figure 3. Solution characterization of amphiphilic nucleobase-containing core-shell BBPs at r.t. ((A) $=$ TBB; $(\mathrm{B})=\mathbf{A B B}$; and $(\mathrm{C})=\mathbf{T B B}+\mathbf{A B B}$, using a 1:1 molar ratio of each). (I) Averaged size distributions (from at least 3 repeat measurements) obtained by DLS for BBPs at $1 \mathrm{mg} \mathrm{mL}^{-1}$ in water. (II) Representative dry-state TEM images obtained from a $1 \mathrm{mg} \mathrm{mL}^{-1}$ solution of BBPs in water and stained with $1 \mathrm{wt} \%$ aqueous uranyl acetate (UA) solution. (III) BBP size distributions along with calculated average diameter values obtained from TEM particle analysis. In each case, at least 100 particles were analyzed.

To evaluate BBP self-assembly driven by complementary non-covalent nucleobase interactions, TBB and ABB solutions were mixed at a 1:1 molar ratio. Surprisingly, no aggregation or assembly was observed by either DLS or TEM upon BBP mixing at room temperature, as shown in Figure 3C. Previous reports on end-to-end assembly of core-shell BBPs demonstrated spontaneous organization into cylindrical nanostructures upon dissolution in aqueous media. ${ }^{35-36}$ The seminal example of dynamic cylindrical 
assembly of BBPs was described by Wooley and coworkers. ${ }^{35}$ In this report, end-to-end assembly was theorized to occur due to strong hydrophobic interactions between styrene units in the core compartments of amphiphilic core-shell BBPs. In this case, BBPs possessed coronae comprised of poly(acrylic acid) (PAA) blocks, which, in neutral water, may not sufficiently stabilize the macromolecules against aggregation. Hierarchical assembly into supramolecular structures would therefore be favored under these conditions to minimize interactions between the polystyrene units and the solvent molecules. In a more recent report by Rzayev and coworkers, end-to-end assembly was observed for BBPs with PAA cores and PEG coronae. ${ }^{36}$ Here, spontaneous self-assembly was driven by the relatively strong H-bonding interactions (in comparison to hydrophobic effects) between PAA domains, which overcame the high shielding tendency of the PEG shells. These assemblies were sensitive to $\mathrm{pH}$ and disassembled upon deprotonation of the acid residues. We initially supposed that the specific H-bonding capacity of the nucleobasecontaining BBPs utilized in our study would promote a similar effect. However, since no spontaneous assembly was observed, even between BBPs with complementary nucleobase functionality, we hypothesized that the PNAM-based corona might effectively inhibit interactions between the BBP cores through steric shielding.

For polymer micelles, steric shielding can often be discouraged by decreasing the solubility of the corona chains in the selected solvent. ${ }^{51}$ In aqueous milieu, this can be accomplished by changing the pH of the solution (in case the corona-forming blocks contain ionizable functionalities), increasing the salt concentration, or by heating. Here, addition of $\mathrm{NaCl}$ to the TBB/ABB mixture did not induce self-assembly. However, a $1 \mathrm{mg} \mathrm{mL}^{-1}$ solution of a 1:1 molar ratio of $\mathbf{T B B} / \mathbf{A B B}$ became turbid upon heating above $70{ }^{\circ} \mathrm{C}$, indicating that self-assembly had occurred.

To investigate the supramolecular assemblies that formed upon heating, DLS analysis and dry-state TEM imaging with sample preparation at elevated temperatures were subsequently conducted. First, a DLS temperature ramp experiment showed a step-wise increase in the size of the dissolved nanostructures that occurred as the solution was heated above $60{ }^{\circ} \mathrm{C}$ (Figure 4A). Although the size values obtained from DLS 
in this experiment were outside of the measurement capacity of the instrument, no precipitation was observed, even at high temperatures. To describe this intriguing self-assembly event in future experiments, the temperature at which this step-change occurs and its magnitude will be considered rather than size values themselves. Second, a sample was prepared by heating a stoichiometric mixture of $\mathbf{T B B}$ and ABB at $70{ }^{\circ} \mathrm{C}$ for $1 \mathrm{~h}$ and then drop-casting this hot sample directly onto a TEM grid prior to imaging. As shown in Figure $4 \mathrm{~B}$, the heated solution contained mixed populations of single bottlebrush molecules and supramolecular cylinders of self-assembled BBPs. Particle size analysis was employed to quantify this distribution, which was conducted on the basis of the area that each particle possessed. A threshold particle area was determined using acquired TEM images of the BBPs in their un-assembled state (e.g., Figures 3AII, 3B-II, and 3C-II). Above this threshold, each particle was considered to be a supramolecular BPP assembly (see Supporting Information for further details). Using this method, the fraction of particle area above the threshold value, which is directly related to the distribution of "unimeric" and assembled BBPs, was determined to be $0.72-0.76$, demonstrating that the majority of the BBPs had undergone self-assembly toward the formation of higher-order supramolecules (Figures 4C and 4D). In addition, the supramolecular BBP assemblies possessed average areas that were at least an order of magnitude larger than the BBPs themselves. This distribution was further confirmed by conducting a DLS temperature ramp experiment under dilute conditions $\left(0.1 \mathrm{mg} \mathrm{mL}^{-1}\right.$ total BBP concentration) (Figure 4E). In this case, intensity-average $D_{\mathrm{h}}$ values and intensity-weighted distributions were within instrumental tolerances, allowing for comparison of the size distributions as a function of temperature. It should be also noted that the onset of transition toward larger supramolecules occurred at higher temperature compared to the TBB/ABB mixture at $1 \mathrm{mg} \mathrm{mL}^{-1}$, owing to a higher input of energy required in this case to compensate for reduced BBPs concentration. Figure $4 \mathrm{~F}$ shows the evolution of intensity-weighted size distributions over the experimentally assessed heating range $\left(25-76^{\circ} \mathrm{C}\right)$. Under these conditions, the relative intensity of the peaks corresponding to "unimers" (black trace) and self-assembled BBPs (purple trace) agreed well with the values obtained from TEM particle analysis, with the assembled BBPs possessing a $D_{\mathrm{h}}$ value that was ca. one order of magnitude larger than the BBP "unimers". 

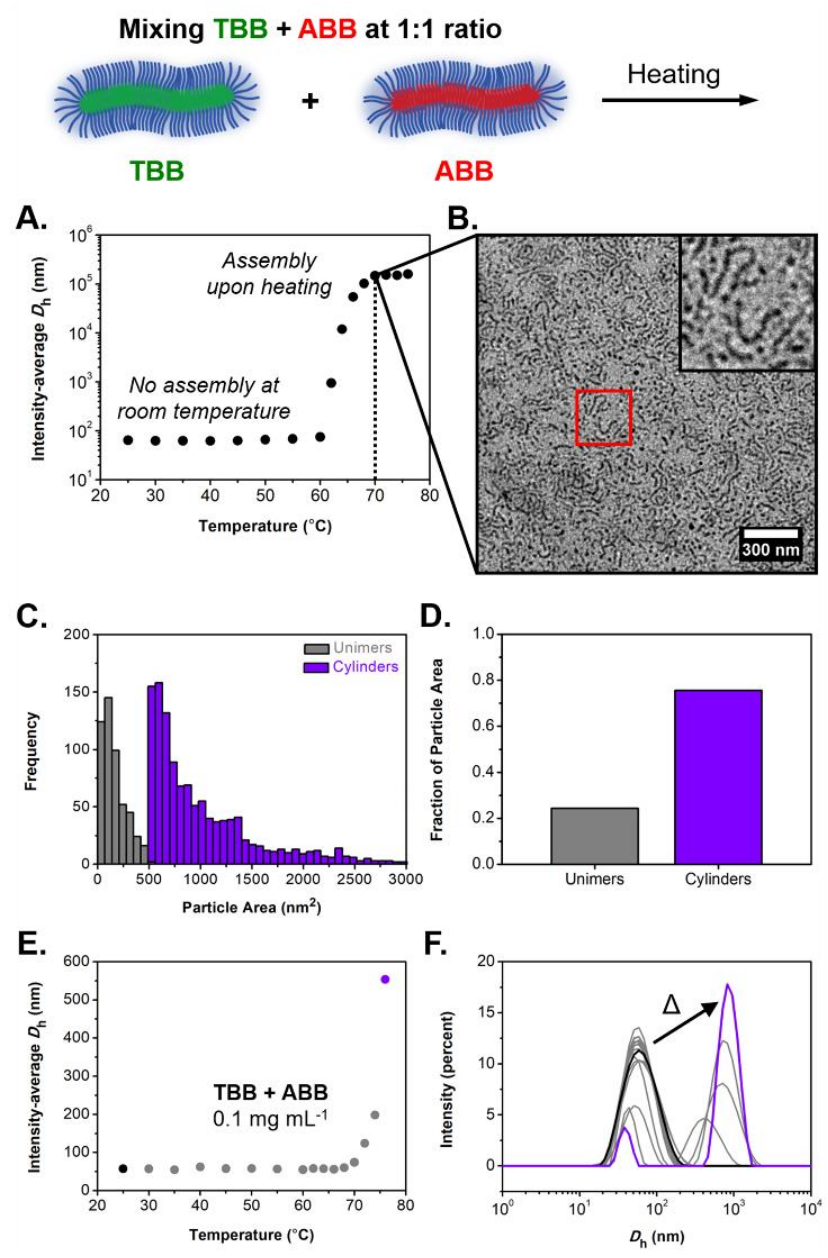

Figure 4. Heating the $\mathbf{T B B} / \mathbf{A B B}$ mixture at elevated temperatures results in the formation of 1D assemblies. (A) Step-change in intensity-average $D_{\mathrm{h}}$ values as a function of solution temperature, as measured by DLS for a $1 \mathrm{mg} \mathrm{mL}^{-1}$ solution of the $\mathbf{T B B} / \mathbf{A B B}$ mixture. (B) Representative dry-state TEM image of the $\mathbf{T B B}+\mathbf{A B B}$ sample $\left(1 \mathrm{mg} \mathrm{mL}^{-1}\right)$ that had been heated at $70{ }^{\circ} \mathrm{C}$ for $1 \mathrm{~h}$ prior to imaging. The grid was prepared by directly drop-casting the heated sample and was stained with $1 \mathrm{wt} \%$ aqueous UA solution. The scale of the inset is $300 \mathrm{~nm} \times 300 \mathrm{~nm}$. (C) Particle analysis based on the acquired TEM image for $\mathbf{T B B} / \mathbf{A B B}$ mixture, quantifying the size of the assemblies with respect to their surface area. (D) Fraction of self-assembled vs. "unimeric" BBPs determined from the particle size analysis in C. (E) Step-change in intensity-average $D_{\mathrm{h}}$ values as a function of temperature, as measured by DLS for a $0.1 \mathrm{mg} \mathrm{mL}^{-1}$ solution of the TBB/ABB mixture. (F) Intensity-weighted size distributions corresponding to the data shown in E. The intensity-average distribution shifts toward larger size values as the temperature increases. 
It is important to note that the cylindrical BBP assemblies obtained by heating the $\mathbf{T B B} / \mathbf{A B B}$ mixture dissociated completely to spherical "unimers" when the solution was allowed to cool to room temperature. Multiple heating and cooling cycles were attempted to drive the self-assembly process toward elongated structures and to stabilize the supramolecules against disassembly. However, as shown in Figure S8, these cycles had an insignificant effect on the size of the BBP assemblies over multiple events. Moreover, "aging" the BBP assemblies by heating a TBB/ABB mixture for a period of $24 \mathrm{~h}$ at $70{ }^{\circ} \mathrm{C}$ also had little influence over their ultimate size or room-temperature stability.

As discussed above, DNA or RNA nucleobases are known to interact in a complementary manner via noncovalent H-bonding. Purines such as thymine (T) (or uracil in RNA) or cytosine (C) are complementary only with the pyrimidines adenine (A) or guanine $(\mathrm{G})$. Non-complementary pairing interactions between purines or pyridines are energetically unfavorable due to overlap repulsion in the former case or overlong $\mathrm{H}$-bond distances in the latter one. ${ }^{52}$ Based on this concept, we hypothesized that nucleobase-containing BBPs would have weak interactions between macromolecules bearing the same bases, but would strongly bind to those containing complementary bases. A series of experiments were designed to evaluate the selectivity of interactions between BBPs containing different nucleobases, or between the BBPs and nucleobase-functionalized linear PNAM- $\boldsymbol{b}$-PTAm and PNAM- $\boldsymbol{b}$-PAAm diblock copolymers prepared via RAFT polymerization, as shown in Figure 5A. First, the largest increase in the size of the BBP assemblies was obtained only when TBB and ABB were mixed stoichiometrically. Solutions of TBB or ABB alone did not exhibit a significant increase in size, even when heated to $>70{ }^{\circ} \mathrm{C}$ (Figure 5B). These data suggest that complementary nucleobase pairing was necessary to form large-scale, stable structures in this system. Second, diblock copolymer $\mathbf{P N A M}_{40}-\boldsymbol{b}-\mathbf{P A A m} \mathbf{m}_{20}\left(\mathbf{A}_{\text {add }}\right)$ was mixed with ABB such that the amount of diblock copolymer was equal to the total number of side-chains of ABB. Similarly, there was no apparent difference in self-assembly behavior upon heating this mixture (Figure 5C). However, when the complementary diblock copolymer $\mathbf{P N A M}_{40}-\boldsymbol{b}$-PTAm 20 ( $\left.\mathbf{T}_{\text {add }}\right)$ was added under otherwise identical 
conditions, the formation of cylindrical BBP assemblies was completely inhibited. It seems to be the case that intermolecular interactions between ABB and $\mathbf{T}_{\text {add }}$ screen further supramolecular BBP-BBP assembly, likely due an increase in corona volume from insertion of the diblock copolymer into the BBP "unimers", which is favored by the relatively stronger H-bonding of the adenine residues of ABB with the complementary bases of $\mathbf{T}_{\text {add }}$ (with respect to A-A H-bonding). This did not occur for $\mathbf{A}_{\text {add, }}$ which further supports the selectivity of complementary nucleobase pairing. Finally, the effect of $\mathbf{T}_{\text {add }}$ on the selfassembly of the $\mathbf{T B B}+\mathbf{A B B}$ mixed system was also investigated. As shown in Figure 5D, addition of $\mathbf{T}_{\text {add }}$ completely prevented the formation of BBP assemblies. The small size of $\mathbf{T}_{\text {add }}$ compared to $\mathbf{T B B}$ likely led to preferential interaction of the diblock copolymer with $\mathbf{A B B}$, limiting interactions between the BBPs themselves. Taken together, these experiments demonstrate that hierarchical self-assembly of nucleobasefunctionalized BBPs, at least in this context, is dominated by complementary base pairing interactions between thymine- and adenine-containing macromolecules.

\section{A. Bottlebrush polymer assembly}

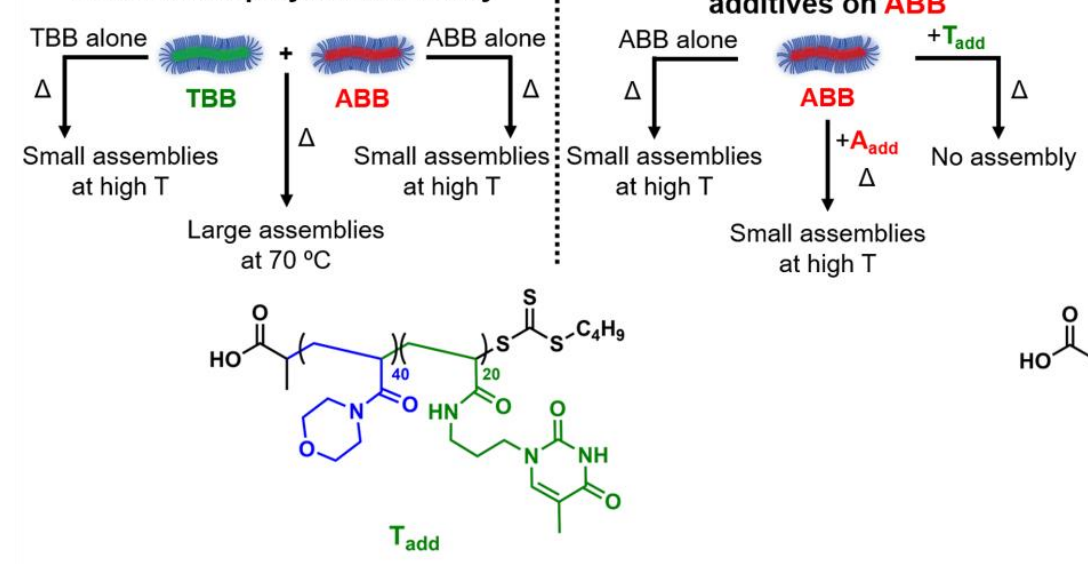

Effect of diblock copolymer additives on $\mathrm{ABB}$

\section{B.}

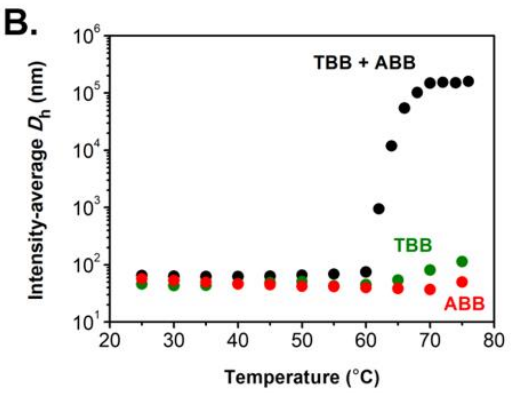

C.

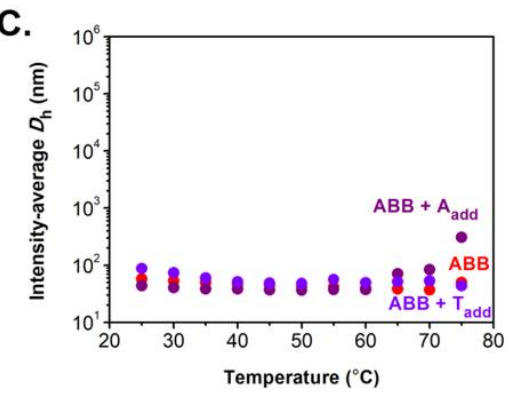

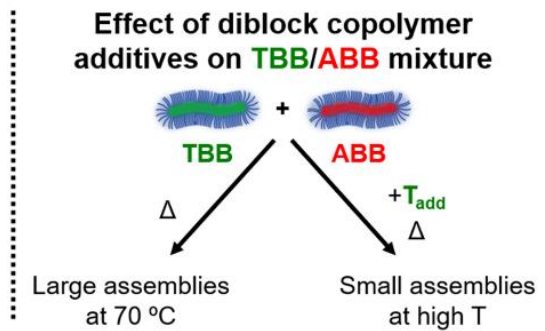

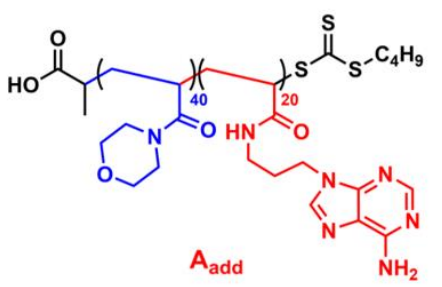

D.

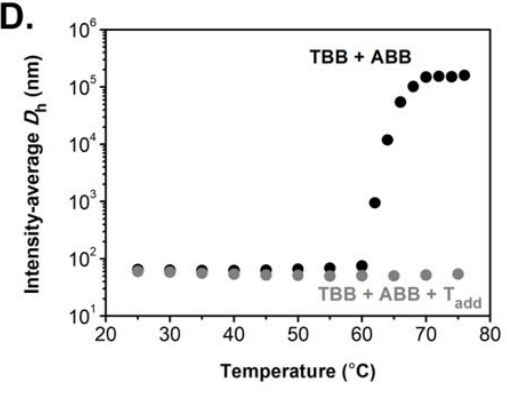


Figure 5. (A) Series of experiments followed for evaluation of selectivity of supramolecular self-assembly of nucleobase-functionalized BBPs. (B) Intensity-average $D_{\mathrm{h}}$ values as a function of temperature for TBB alone, ABB alone, and the TBB + ABB mixture, as measured by DLS. The largest assemblies were obtained when both TBB and ABB were present. (C) Addition of non-complementary $\mathbf{A}_{\text {add }}$ diblock copolymer, containing AAm units, does not appear to influence self-assembly, whilst $\mathbf{T}_{\text {add }}$ diblock copolymer containing complementary functionality (TAm units) prevents self-assembly of ABB. (D) Addition of a complementary $\mathbf{T}_{\text {add }}$ diblock copolymer, containing TAm repeat units, discourages self-assembly between TBB and ABB. The total BBP concentration was $1 \mathrm{mg} \mathrm{mL}^{-1}$ in all cases.

Self-assembly of the BBPs prepared herein toward formation of cylindrical supramolecules only occurred once the samples were heated above a certain threshold ( $c a .60{ }^{\circ} \mathrm{C}$ for the $\mathbf{T B B} / \mathbf{A B B}$ mixed sample). This thermally-dependent behavior was hypothesized to originate from reduced shielding by the corona-forming chains at elevated temperatures, leading to the formation of supramolecular $\mathbf{T B B}+\mathbf{A B B}$ chains. The most reasonable explanation for this phenomenon is the existence of a lower critical solution temperature (LCST) transition for PNAM chains in the BBP shells. Linear PNAM is not known to possess LCST behavior in aqueous media (Figure S10). ${ }^{53}$ However, numerous reports have demonstrated that LCST varies with polymer topology, with graft polymers generally exhibiting lower LCSTs than their linear counterparts. The close packing of polymer chains in a densely-grafted polymer (i.e., in BBPs) facilitates the exclusion of water upon heating, lowering the temperature at which this transition occurs. Thus, we wondered whether BBPs with PNAM homopolymer side-chains would conform to this trend; therefore, a NB-PNAM 38 macromonomer was prepared via RAFT polymerization of NAM using a norbornene-functionalized chain transfer agent (Table S3). BBP synthesis using the resulting macromonomer was then achieved via ROMP "grafting-through" polymerization, as shown in Figure 6A, to produce a series of $\mathbf{P N B}_{\mathbf{n}}-\mathbf{g}-\mathbf{P N A M} \mathbf{M}_{\mathbf{3 8}}(\mathrm{n}=$ $15,30,45,60,75)$ BBPs with increasing backbone degrees of polymerization and low $\bigoplus_{\mathrm{M}}$ values (Figures 6B and 6C, and Table S4). LCST values for these BBPs were then determined using variable temperature UV-Vis spectroscopy, where in each case the LCST was taken to be the inflection point of the obtained 
absorbance vs. temperature curve. Sharp LCST transitions were observed for each of the five BBPs prepared, and the LCST values were found to decrease with increasing BBP molecular weight (Figure 6D). In addition, the dependence of LCST on BBP molecular weight agreed well with the trend predicted by the Flory-Huggins combinatorial entropy approximation, LCST $\propto 1 / 2\left(1+\mathrm{MW}^{-2}\right)^{2},{ }^{54}$ confirming that this transition did indeed occur from an entropically-favored exclusion of water molecules from the solvated BBPs (Figure 6E). Whilst LCST values for nucleobase-containing BBPs could not be easily determined using UV-Vis spectroscopy due to coincidence of LCST and self-assembly phenomena, we assumed that the PNAM blocks in the amphiphilic core-shell BBPs also experience molecular weight-dependent LCST transitions similar to block copolymers with LCST polymers in their hydrophilic domains. ${ }^{55}$
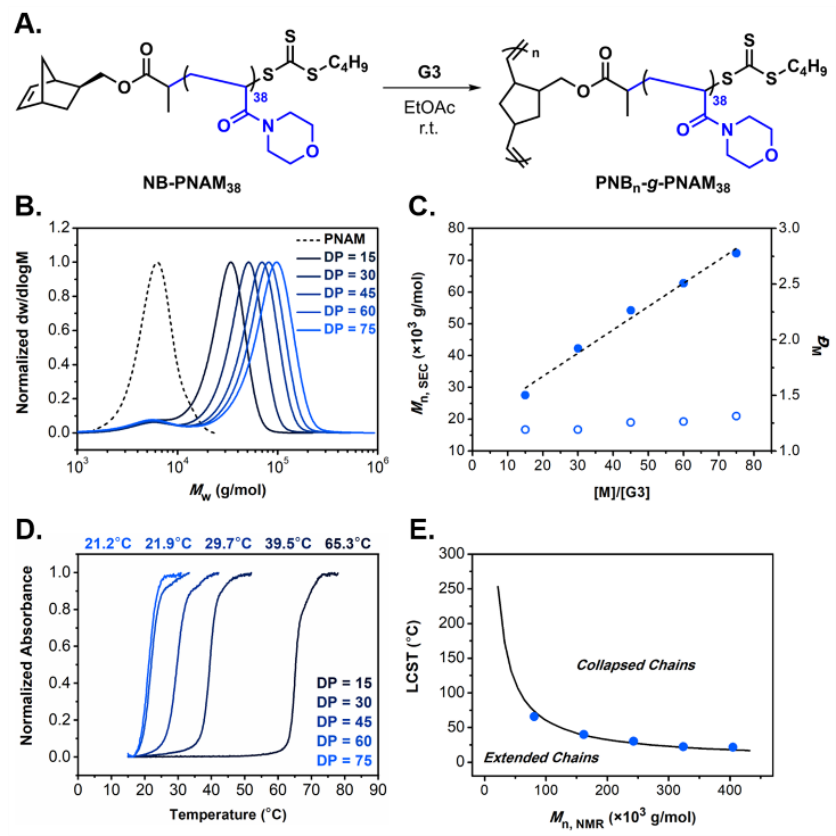

Figure 6. Change in LCST as a function of backbone DP for $\mathbf{P N B}_{\mathbf{n}} \mathbf{- g}-\mathbf{P N A M} \mathbf{M}_{38}$ BBPs. (A) Synthesis of PNB $_{\text {- }}$ g-PNAM 38 BBPs via ROMP "grafting-through" using G3. (B) Normalized SEC RI molecular weight distributions of NB-PNAM 38 macromonomer (dashed line) and prepared $\mathbf{P N B}_{\mathbf{n}} \mathbf{- g}-\mathbf{P N A M} \mathbf{M M}_{38}$ BBPs with increasing backbone DPs (solid lines). (C) Evolution of $M_{\mathrm{n}}$ (filled circles) and $\bigoplus_{\mathrm{M}}$ (empty circles) values with increasing targeted backbone DP calculated from SEC analysis. Reported $M_{\mathrm{n}}$ and $\bigoplus_{\mathrm{M}}$ values were calculated from PMMA standards using $\mathrm{DMF}+5 \mathrm{mM} \mathrm{NH}_{4} \mathrm{BF}_{4}$ as the eluent. (D) Variable temperature 
absorbance spectroscopy curves and corresponding LCST values of $\mathbf{P N B}_{\mathbf{n}}$ - $\boldsymbol{g}$-PNAM $\mathbf{P A}_{38}$ BBPs. (E) Measured LCST values as a function of BBP number-average molecular weight $\left(M_{\mathrm{n}}\right)$. The LCST values decrease with increasing BBP molecular weight. The solid line represents theoretical LCST values predicted by a modification of the Flory-Huggins combinatorial entropy approximation: $\mathrm{LCST} \propto 1 / 2\left(1+\mathrm{MW}^{-2}\right)^{2}$.

Based on the selectivity and temperature dependence of the self-assembly process of the developed nucleobase-containing BBPs, we hypothesized that the formation of supramolecular BBP structures occurred according to the mechanism proposed in Figure 7A. At room temperature, steric shielding interactions of the PNAM coronae prevented the formation of large scale assemblies (although dimeric and other smaller species likely exist in equilibrium with unimers, as discussed previously) (Figure 7B). Upon heating, the BBPs experienced an LCST transition in their coronae (Figures 6D and 6E), which resulted in both increased hydrophobicity of the BBP "unimers" and exposure of core-block interfaces at the BBP chain-ends. This was also supported by DLS and static light scattering (SLS) measurements of the TBB + ABB mixture as a function of solution temperature. As shown in Figure 7C, $R_{\mathrm{h}}$ values of the core-shell BBPs initially decreased with increasing temperature, whereas $R_{\mathrm{g}}$ values remained relatively constant, suggesting that the extent of stretching of the corona-forming chains progressively decreased resulting in thinner and more compact structures ${ }^{56}$ Moreover, a concurrent $R_{\mathrm{g}} / R_{\mathrm{h}}$ ratio increase was also determined upon increasing solution temperature, suggesting the formation of more anisotropic rod-like structures (Figure S9). ${ }^{57}$ Above a critical temperature, hierarchical self-assembly allowed for formation of long, 1D cylindrical supramolecules of BBPs (Figure 7B). This supramolecular polymerization process is thought to occur via an isodesmic mechanism, wherein TBB and ABB "unimers" were incorporated in an alternating fashion. ${ }^{58-59}$ This proposed mechanism is evidenced by: (1) the sensitivity of the assembly process to nucleobase-chemistry (Figure 5); (2) the strong dependence of the assembly process on concentration (Figure 7D); and (3) the dramatic influence of $\mathbf{T B B} / \mathbf{A B B}$ stoichiometry (Figure 7E), consistent with an alternating incorporation of BBP amphiphiles. It should be noted that the distribution of BBP "unimers" 
and assemblies could not be driven toward a single population of pure self-assembled supramolecules. Whilst heating the BBP samples resulted in increased hydrophobicity, which favored self-assembly, it also likely decreased the strength of H-bonding interactions between nucleobases (H-bond strength/length is exquisitely sensitive to temperature), acting to drive the equilibrium back toward the BBP "unimers".

A.

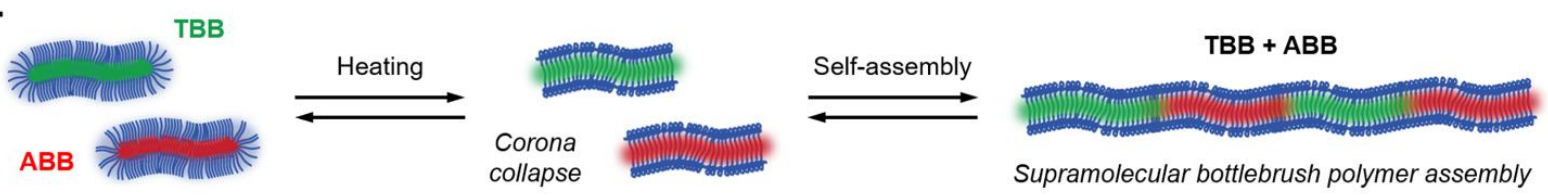

B.
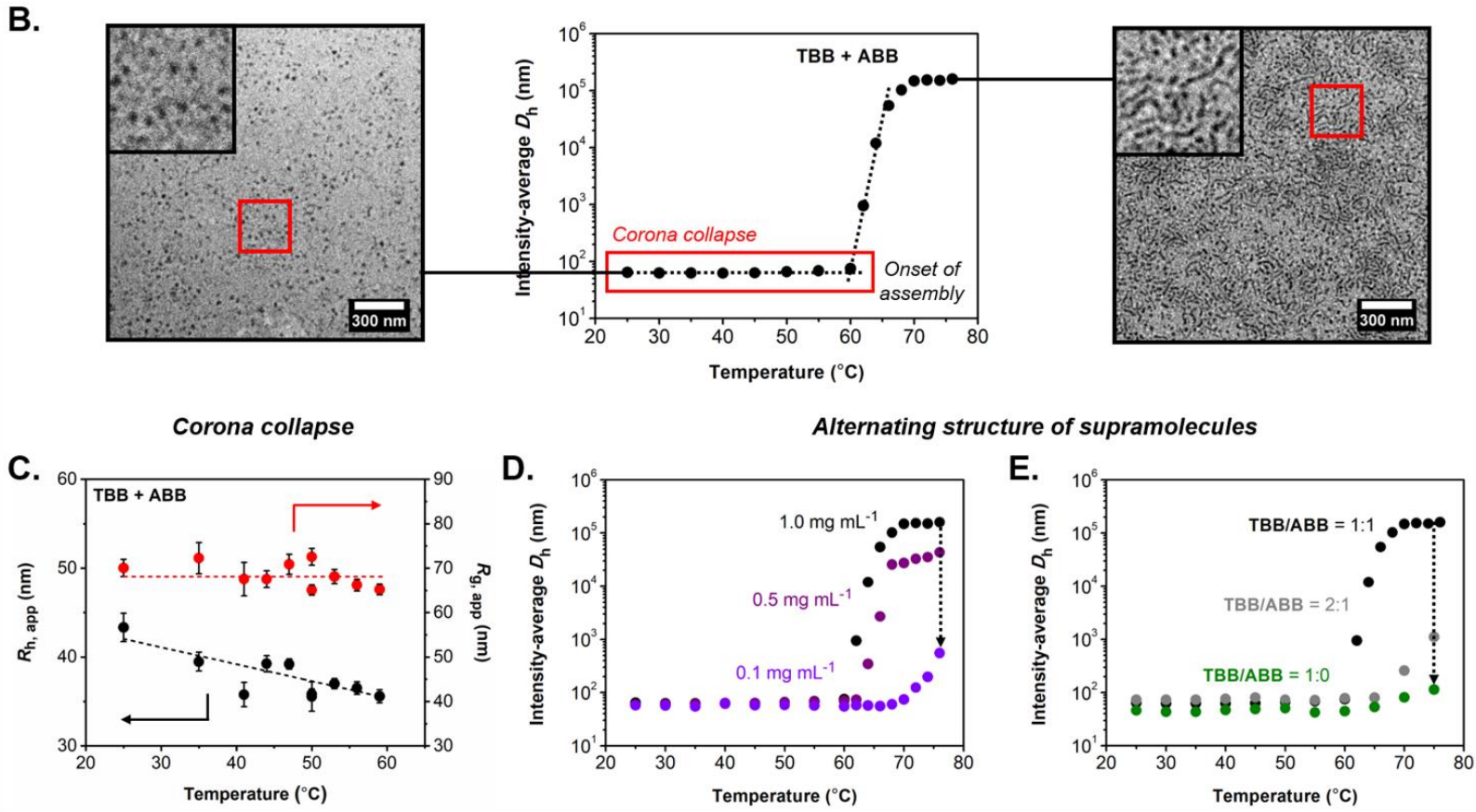

Figure 7. Proposed isodesmic mechanism for formation of supramolecular nucleobase-containing BBP assemblies. (A) Heating the $\mathbf{T B B} / \mathbf{A B B}$ mixture at elevated temperatures results in collapse of the PNAM corona chains and exposure of core-block interfaces at the BBP chain-ends, increasing the hydrophobicity of the BBP "unimers". To compensate for an increased energy of interaction with the solvent, the BBPs assemble into supramolecular chains of alternating $\mathbf{T B B}$ and $\mathbf{A B B}$ units. (B) Step-change in intensityaverage $D_{\mathrm{h}}$ values as a function of solution temperature, as measured by DLS for a $1 \mathrm{mg} \mathrm{mL}^{-1}$ solution of the $\mathbf{T B B} / \mathbf{A B B}$ mixture in water, and representative dry-state TEM images of the $\mathbf{T B B} / \mathbf{A B B}$ sample at room temperature and upon heating at $70{ }^{\circ} \mathrm{C}$ for $1 \mathrm{~h}$ prior to imaging. In both cases, TEM grids were stained with 
$1 \mathrm{wt} \%$ aqueous UA solution. The scale of the insets is $300 \mathrm{~nm} \times 300 \mathrm{~nm}$. (C) Change in $R_{\mathrm{h}}$ (black circles) and $R_{\mathrm{g}}$ (red circles) values with increasing solution temperature, as determined by DLS and SLS for a 0.1 $\mathrm{mg} \mathrm{mL} \mathrm{m}^{-1}$ solution of the $\mathbf{T B B} / \mathbf{A B B}$ mixture, supporting the corona collapse hypothesis, as BBPs progressively become thinner and more compact. (D) The alternating structure arises from an isodesmic mechanism of assembly. The step-change in intensity-average $D_{\mathrm{h}}$ as a function of temperature becomes less dramatic at lower BBP concentrations, which is a key characteristic of isodesmic supramolecular polymerizations. (E) A dramatic dependence of the magnitude of the step-change in intensity-average $D_{\mathrm{h}}$ on the stoichiometry of the TBB/ABB mixture further supports addition of BBP "unimers" in an alternating manner.

In an attempt to maintain the morphology of the supramolecular $\mathbf{T B B}+\mathbf{A B B}$ assemblies upon cooling their solution to room temperature, a covalent cross-linking methodology was subsequently utilized. In particular, the aqueous solution of the $\mathbf{T B B} / \mathbf{A B B}$ mixture at $1: 1$ molar ratio was first heated at $70{ }^{\circ} \mathrm{C}$ to promote their higher-order assembly and then $N, N^{\prime}$-methylenebis(acrylamide) (MBAm) and radical initiator (V-50) were added resulting in inter- and intramolecular cross-linking via RAFT polymerization, yielding corona-cross-linked supramolecules (Figure 8A). The resulting solution was subsequently cooled to room temperature and the cross-linking efficiency was evaluated upon comparison of the relative fraction between self-assembled and "unimeric" BBPs from acquired TEM images (Figures 8B and 8E). On the basis of TEM image analysis and contrary to TBB/ABB sample heated at elevated temperatures (Figures 8C and 8D), it is evident that the majority of supramolecular BBP chains disassembled into individual coreshell "unimers", whereas only a small population $(\sim 26 \%)$ of cylindrical assemblies was present for the sample that was cooled to room temperature (Figures 8F and 8G). Intriguingly, no gelation or macroscopic aggregation was observed during cross-linking, further supporting the proposed selective end-to-end assembly phenomenon. The relatively poor cross-linking efficiency achieved in this experiment could have resulted from the loss of CTA end groups during RAFT "grafting-from" or to decomposition during the initial self-assembly process. It could also be the case that cross-linking, and thus cylinder retention, could 
have been enhanced by the use of a cross-linker with a longer methylene spacer. In any case, this experiment highlights both the transient nature of the end-to-end bottlebrush assembly mechanism in this system and the potential for the selective development of long, cylindrical supramolecules through sequential hierarchical assembly and cross-linking steps.

A.

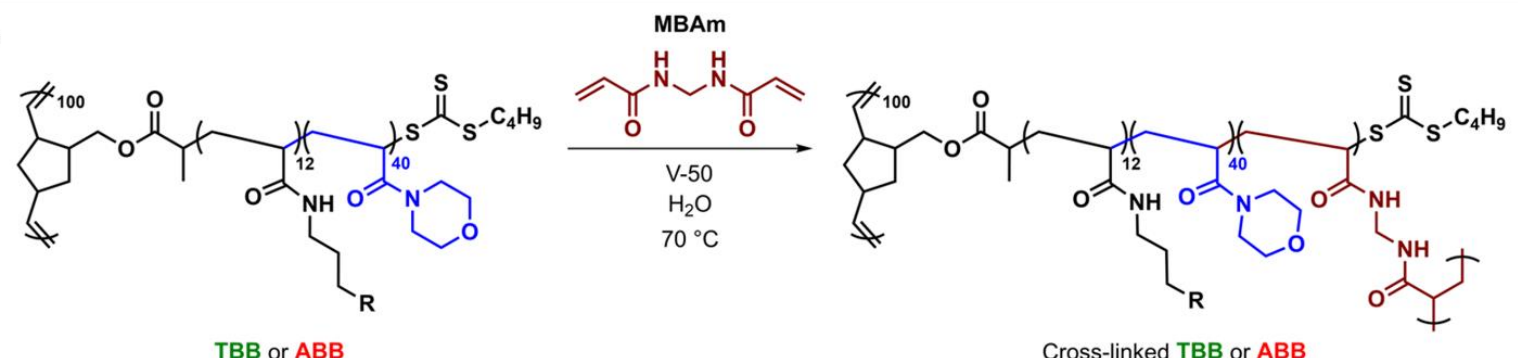

B.

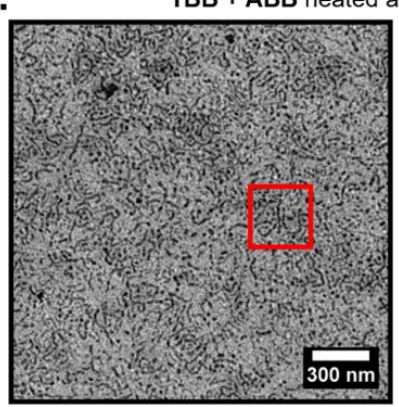

C.

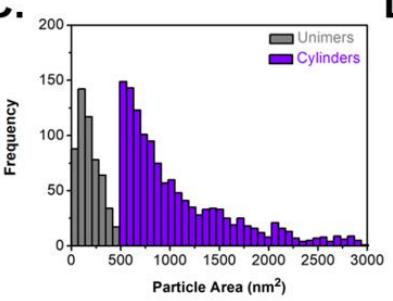

D.

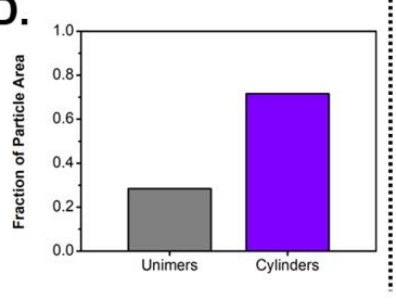

E.
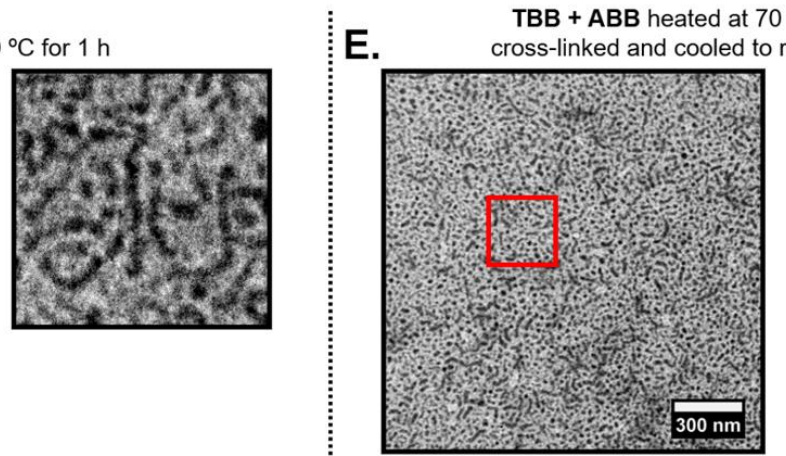

F.

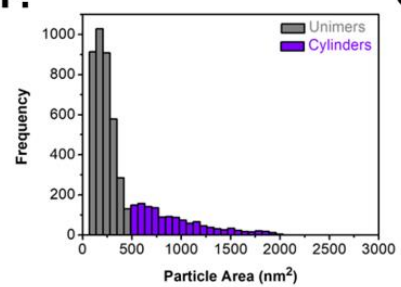

G.

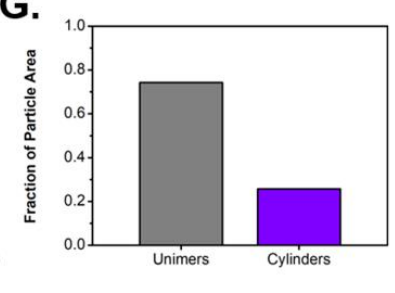

Figure 8. Cross-linking of the supramolecular BBP assemblies at $70{ }^{\circ} \mathrm{C}$ for evaluation of morphology retention at room temperature. (A) Cross-linking was carried out in the presence of $N, N^{\prime}-$ methylenebis(acrylamide) (MBAm) that led to the formation of covalently stabilized assemblies. (B) Representative dry-state TEM image of the $\mathbf{T B B}+\mathbf{A B B}$ sample $\left(1 \mathrm{mg} \mathrm{mL}^{-1}\right)$ that had been heated at $70{ }^{\circ} \mathrm{C}$ for $1 \mathrm{~h}$ prior to imaging. The grid was prepared by directly drop-casting the heated sample and was stained with $1 \mathrm{wt} \%$ aqueous UA solution. The scale of the enlarged region is $300 \times 300 \mathrm{~nm}^{2}$. (C) Particle analysis based on the acquired TEM image for heated $\mathbf{T B B}+\mathbf{A B B}$ sample at $70{ }^{\circ} \mathrm{C}$, quantifying the size of the 
assemblies with respect to their surface area. (D) Fraction of self-assembled vs. "unimeric" BBPs determined from the particle size analysis in C. (E) Representative dry-state TEM image of the $\mathbf{T B B}+\mathbf{A B B}$ sample $\left(1 \mathrm{mg} \mathrm{mL}^{-1}\right)$ that had been heated at $70{ }^{\circ} \mathrm{C}$ for $1 \mathrm{~h}$, cross-linked with MBAm at $70{ }^{\circ} \mathrm{C}$, and then cooled to room temperature prior to imaging. The grid was stained with $1 \mathrm{wt} \%$ aqueous UA solution. The scale of the enlarged region is $300 \mathrm{~nm} \times 300 \mathrm{~nm}$. (F) Particle analysis based on the acquired TEM image for cross-linked $\mathbf{T B B}+\mathbf{A B B}$ sample, quantifying the size of the assemblies with respect to their surface area. (G) Fraction of self-assembled vs. "unimeric" BBPs determined from the particle size analysis in F.

\section{CONCLUSIONS}

In summary, the synthesis and self-assembly of nucleobase-containing amphiphilic "core-shell" bottlebrush polymers in solution were presented herein. To the best of our knowledge, this report represents the first evidence of thermally-induced BBP supramolecular assembly mediated by the development of complementary nucleobase interactions. We also provided evidence for a mechanism of assembly mediated by the thermally-induced collapse of the stabilizing corona blocks, facilitating close interactions between functional groups at the BBP chain-ends. Due to the high selectivity of nucleobase interactions, this selfassembly behavior was only observed when equimolar amounts of BBPs containing complementary thymine and adenine functionalities were mixed and heated at elevated temperatures. The evident sensitivity of this supramolecular assembly procedure to both stoichiometry and concentration implied that an alternating, isodesmic mechanism was in operation. This report highlights the unique asymmetric reactivity of BBPs and informs on the importance of steric shielding in core-shell BBP-based architectures on the growth and disassembly of cylindrical supramolecules. Further, it provides additional insight on how polymer topology influences the physical properties of the polymeric side-chains through the introduction of LCST behavior to a polymer that typically does not exhibit thermally-dependent solubility in its linear form. The discoveries reported herein can be leveraged in future studies to rationally design stimuliresponsive, dynamic bottlebrush assemblies. 


\section{ASSOCIATED CONTENT}

Supporting Information. The Supporting Information is available free of charge on the ACS Publications website at DOI:

Materials and characterization techniques, synthetic methods, supplementary NMR and SEC analysis data of BBPs, supplementary DLS and SLS data of amphiphilic BBP-based assemblies (PDF)

\section{AUTHOR INFORMATION}

\section{Corresponding Authors}

* Email: j.c.foster@bham.ac.uk (J.C.F.)

* Email: r.oreilly@bham.ac.uk (R.K.O.R.)

\section{Author Contributions}

${ }^{\nabla S}$.V. and Z.H. contributed equally to this work. The manuscript was written through contributions of all authors. All authors have given approval to the final version of the manuscript.

\section{Notes}

The authors declare no competing financial interest.

\section{ACKNOWLEDGMENTS}

This work was supported by the ERC (grant number 615142), the University of Birmingham, and the University of Warwick. Dr. Z. Hua acknowledges China Scholarship Council (CSC) for funding.

\section{REFERENCES}

1. Philp, D.; Stoddart, J. F., Self-Assembly in Natural and Unnatural Systems. Angew. Chem. Int. Ed. 1996, 35, 1154-1196.

2. Mendes, A. C.; Baran, E. T.; Reis, R. L.; Azevedo, H. S., Self-assembly in nature: using the principles of nature to create complex nanobiomaterials. WIREs Nanomed. Nanobiotechnol. 2013, 5, 582-612.

3. Whitford, D., Proteins: Structure and Function. Wiley: 2013. 
4. Mart, R. J.; Osborne, R. D.; Stevens, M. M.; Ulijn, R. V., Peptide-based stimuli-responsive biomaterials. Soft Matter 2006, 2, 822-835.

5. Pieters, B. J. G. E.; van Eldijk, M. B.; Nolte, R. J. M.; Mecinović, J., Natural supramolecular protein assemblies. Chem. Soc. Rev. 2016, 45, 24-39.

6. Fukata, Y.; Itoh, T. J.; Kimura, T.; Ménager, C.; Nishimura, T.; Shiromizu, T.; Watanabe, H.; Inagaki, N.; Iwamatsu, A.; Hotani, H.; Kaibuchi, K., CRMP-2 binds to tubulin heterodimers to promote microtubule assembly. Nat. Cell Biol. 2002, 4, 583-591.

7. Foster, J. C.; Varlas, S.; Couturaud, B.; Coe, Z.; O'Reilly, R. K., Getting into Shape: Reflections on a New Generation of Cylindrical Nanostructures' Self-Assembly Using Polymer Building Blocks. J. Am. Chem. Soc. 2019, 141, 2742-2753.

8. Otter, R.; Besenius, P., Supramolecular assembly of functional peptide-polymer conjugates. Org. Biomol. Chem. 2019, 17, 6719-6734.

9. Gilroy, J. B.; Gädt, T.; Whittell, G. R.; Chabanne, L.; Mitchels, J. M.; Richardson, R. M.; Winnik, M. A.; Manners, I., Monodisperse cylindrical micelles by crystallization-driven living self-assembly. Nat. Chem. 2010, 2, 566-570.

10. Arno, M. C.; Inam, M.; Coe, Z.; Cambridge, G.; Macdougall, L. J.; Keogh, R.; Dove, A. P.; O’Reilly, R. K., Precision Epitaxy for Aqueous 1D and 2D Poly(E-caprolactone) Assemblies. J. Am. Chem. Soc. 2017, 139, 16980-16985.

11. Choi, I.; Yang, S.; Choi, T.-L., Preparing Semiconducting Nanoribbons with Tunable Length and Width via Crystallization-Driven Self-Assembly of a Simple Conjugated Homopolymer. J. Am. Chem. Soc. 2018, 140, 17218-17225.

12. Cui, H.; Webber, M. J.; Stupp, S. I., Self-assembly of peptide amphiphiles: From molecules to nanostructures to biomaterials. J. Pept. Sci. 2010, 94, 1-18.

13. Hendricks, M. P.; Sato, K.; Palmer, L. C.; Stupp, S. I., Supramolecular Assembly of Peptide Amphiphiles. Acc. Chem. Res. 2017, 50, 2440-2448.

14. Couet, J.; Samuel, J. D. J. S.; Kopyshev, A.; Santer, S.; Biesalski, M., Peptide-Polymer Hybrid Nanotubes. Angew. Chem. Int. Ed. 2005, 44, 3297-3301.

15. Hourani, R.; Zhang, C.; van der Weegen, R.; Ruiz, L.; Li, C.; Keten, S.; Helms, B. A.; Xu, T., Processable Cyclic Peptide Nanotubes with Tunable Interiors. J. Am. Chem. Soc. 2011, 133, 15296-15299. 16. Rho, J. Y.; Cox, H.; Mansfield, E. D. H.; Ellacott, S. H.; Peltier, R.; Brendel, J. C.; Hartlieb, M.; Waigh, T. A.; Perrier, S., Dual self-assembly of supramolecular peptide nanotubes to provide stabilisation in water. Nat. Commun. 2019, 10, 4708.

17. Schappacher, M.; Deffieux, A., Synthesis of Macrocyclic Copolymer Brushes and Their Self-Assembly into Supramolecular Tubes. Science 2008, 319, 1512-1515.

18. Sato, K.; Itoh, Y.; Aida, T., Homochiral supramolecular polymerization of bowl-shaped chiral macrocycles in solution. Chem. Sci. 2014, 5, 136-140.

19. Heo, J.-M.; Kim, Y.; Han, S.; Joung, J. F.; Lee, S.-h.; Han, S.; Noh, J.; Kim, J.; Park, S.; Lee, H.; Choi, Y. M.; Jung, Y.-S.; Kim, J.-M., Chromogenic Tubular Polydiacetylenes from Topochemical Polymerization of Self-Assembled Macrocyclic Diacetylenes. Macromolecules 2017, 50, 900-913.

20. Messmore, B. W.; Hulvat, J. F.; Sone, E. D.; Stupp, S. I., Synthesis, Self-Assembly, and Characterization of Supramolecular Polymers from Electroactive Dendron Rodcoil Molecules. J. Am. Chem. Soc. 2004, 126, 14452-14458.

21. Dong, R.; Zhou, Y.; Zhu, X., Supramolecular Dendritic Polymers: From Synthesis to Applications. Acc. Chem. Res. 2014, 47, 2006-2016.

22. De Greef, T. F. A.; Smulders, M. M. J.; Wolffs, M.; Schenning, A. P. H. J.; Sijbesma, R. P.; Meijer, E. W., Supramolecular Polymerization. Chem. Rev. 2009, 109, 5687-5754.

23. Fox, J. D.; Rowan, S. J., Supramolecular Polymerizations and Main-Chain Supramolecular Polymers. Macromolecules 2009, 42, 6823-6835.

24. Paul, B. D.; Sbodio, J. I.; Xu, R.; Vandiver, M. S.; Cha, J. Y.; Snowman, A. M.; Snyder, S. H., Cystathionine $\gamma$-lyase deficiency mediates neurodegeneration in Huntington's disease. Nature 2014, 509, 96-100. 
25. Aida, T.; Meijer, E. W.; Stupp, S. I., Functional Supramolecular Polymers. Science 2012, 335, 813-817. 26. Insua, I.; Montenegro, J., 1D to 2D Self Assembly of Cyclic Peptides. J. Am. Chem. Soc. 2020, 142, 300-307.

27. Grubbs, R. B.; Grubbs, R. H., 50th Anniversary Perspective: Living Polymerization-Emphasizing the Molecule in Macromolecules. Macromolecules 2017, 50, 6979-6997.

28. Sheiko, S. S.; Sumerlin, B. S.; Matyjaszewski, K., Cylindrical molecular brushes: Synthesis, characterization, and properties. Prog. Polym. Sci. 2008, 33, 759-785.

29. Verduzco, R.; Li, X.; Pesek, S. L.; Stein, G. E., Structure, function, self-assembly, and applications of bottlebrush copolymers. Chem. Soc. Rev. 2015, 44, 2405-2420.

30. Alaboalirat, M.; Qi, L.; Arrington, K. J.; Qian, S.; Keum, J. K.; Mei, H.; Littrell, K. C.; Sumpter, B. G.; Carrillo, J.-M. Y.; Verduzco, R.; Matson, J. B., Amphiphilic Bottlebrush Block Copolymers: Analysis of Aqueous Self-Assembly by Small-Angle Neutron Scattering and Surface Tension Measurements. Macromolecules 2019, 52, 465-476.

31. Fenyves, R.; Schmutz, M.; Horner, I. J.; Bright, F. V.; Rzayev, J., Aqueous Self-Assembly of Giant Bottlebrush Block Copolymer Surfactants as Shape-Tunable Building Blocks. J. Am. Chem. Soc. 2014, 136, 7762-7770.

32. Cheng, L.-C.; Gadelrab, K. R.; Kawamoto, K.; Yager, K. G.; Johnson, J. A.; Alexander-Katz, A.; Ross, C. A., Templated Self-Assembly of a PS-Branch-PDMS Bottlebrush Copolymer. Nano Lett. 2018, 18, 4360-4369.

33. Miyake, G. M.; Piunova, V. A.; Weitekamp, R. A.; Grubbs, R. H., Precisely Tunable Photonic Crystals From Rapidly Self-Assembling Brush Block Copolymer Blends. Angew. Chemie. Int. Ed. 2012, 51, 1124611248.

34. Sveinbjörnsson, B. R.; Weitekamp, R. A.; Miyake, G. M.; Xia, Y.; Atwater, H. A.; Grubbs, R. H., Rapid self-assembly of brush block copolymers to photonic crystals. Proc. Natl. Acad. Sci. U.S.A. 2012, 109, 14332-14336.

35. Li, Z.; Ma, J.; Lee, N. S.; Wooley, K. L., Dynamic Cylindrical Assembly of Triblock Copolymers by a Hierarchical Process of Covalent and Supramolecular Interactions. J. Am. Chem. Soc. 2011, 133, 12281231.

36. Onbulak, S.; Rzayev, J., Synthesis and one-dimensional assembly of cylindrical polymer nanoparticles prepared from tricomponent bottlebrush copolymers. J. Poly. Sci. Part A: Polym. Chem. 2017, 55, 38683874.

37. Sivakova, S.; Rowan, S. J., Nucleobases as supramolecular motifs. Chem. Soc. Rev. 2005, 34, 9-21.

38. Spijker, H. J.; Dirks, A. J.; van Hest, J. C. M., Synthesis and assembly behavior of nucleobasefunctionalized block copolymers. J. Poly. Sci. Part A: Polym. Chem. 2006, 44, 4242-4250.

39. Fathalla, M.; Lawrence, C. M.; Zhang, N.; Sessler, J. L.; Jayawickramarajah, J., Base-pairing mediated non-covalent polymers. Chem. Soc. Rev. 2009, 38, 1608-1620.

40. McHale, R.; O'Reilly, R. K., Nucleobase Containing Synthetic Polymers: Advancing Biomimicry via Controlled Synthesis and Self-Assembly. Macromolecules 2012, 45, 7665-7675.

41. Kang, Y.; Pitto-Barry, A.; S. Rolph, M.; Hua, Z.; Hands-Portman, I.; Kirby, N.; O'Reilly, R. K., Use of complementary nucleobase-containing synthetic polymers to prepare complex self-assembled morphologies in water. Polym. Chem. 2016, 7, 2836-2846.

42. Hua, Z.; Pitto-Barry, A.; Kang, Y.; Kirby, N.; Wilks, T. R.; O'Reilly, R. K., Micellar nanoparticles with tuneable morphologies through interactions between nucleobase-containing synthetic polymers in aqueous solution. Polym. Chem. 2016, 7, 4254-4262.

43. Hua, Z.; Keogh, R.; Li, Z.; Wilks, T. R.; Chen, G.; O’Reilly, R. K., Reversibly Manipulating the Surface Chemistry of Polymeric Nanostructures via a "Grafting To" Approach Mediated by Nucleobase Interactions. Macromolecules 2017, 50, 3662-3670.

44. Hua, Z.; Wilks, T. R.; Keogh, R.; Herwig, G.; Stavros, V. G.; O'Reilly, R. K., Entrapment and Rigidification of Adenine by a Photo-Cross-Linked Thymine Network Leads to Fluorescent Polymer Nanoparticles. Chem. Mater. 2018, 30, 1408-1416. 
45. Hua, Z.; Jones, J. R.; Thomas, M.; Arno, M. C.; Souslov, A.; Wilks, T. R.; O’Reilly, R. K., Anisotropic polymer nanoparticles with controlled dimensions from the morphological transformation of isotropic seeds. Nat. Commun. 2019, 10, 5406.

46. Pelegri-O'Day, E. M.; Lin, E.-W.; Maynard, H. D., Therapeutic Protein-Polymer Conjugates: Advancing Beyond PEGylation. J. Am. Chem. Soc. 2014, 136, 14323-14332.

47. Aragón, S. R.; Pecora, R., Theory of dynamic light scattering from polydisperse systems. J. Chem. Phys. 1976, 64, 2395-2404.

48. Zhang, Z.; Carrillo, J.-M. Y.; Ahn, S.-k.; Wu, B.; Hong, K.; Smith, G. S.; Do, C., Atomistic Structure of Bottlebrush Polymers: Simulations and Neutron Scattering Studies. Macromolecules 2014, 47, 58085814.

49. Pesek, S. L.; Xiang, Q.; Hammouda, B.; Verduzco, R., Small-angle neutron scattering analysis of bottlebrush backbone and side chain flexibility. J. Polym. Sci., Part B: Polym. Phys. 2017, 55, 104-111.

50. Gallyamov, M. O.; Tartsch, B.; Mela, P.; Potemkin, I. I.; Sheiko, S. S.; Börner, H.; Matyjaszewski, K.; Khokhlov, A. R.; Möller, M., Vapor-induced spreading dynamics of adsorbed linear and brush-like macromolecules as observed by environmental SFM: Polymer chain statistics and scaling exponents. $J$. Polym. Sci., Part B: Polym. Phys. 2007, 45, 2368-2379.

51. Mai, Y.; Eisenberg, A., Self-assembly of block copolymers. Chem. Soc. Rev. 2012, 41, 5969-5985.

52. Yakovchuk, P.; Protozanova, E.; Frank-Kamenetskii, M. D., Base-stacking and base-pairing contributions into thermal stability of the DNA double helix. Nucleic acids res. 2006, 34, 564-574.

53. Eggenhuisen, T. M.; Becer, C. R.; Fijten, M. W. M.; Eckardt, R.; Hoogenboom, R.; Schubert, U. S., Libraries of Statistical Hydroxypropyl Acrylate Containing Copolymers with LCST Properties Prepared by NMP. Macromolecules 2008, 41, 5132-5140.

54. Patterson, D., Free Volume and Polymer Solubility. A Qualitative View. Macromolecules 1969, 2, 672677.

55. Blackman, L. D.; Gibson, M. I.; O'Reilly, R. K., Probing the causes of thermal hysteresis using tunable Nagg micelles with linear and brush-like thermoresponsive coronas. Polym. Chem. 2017, 8, 233-244.

56. Zhou, H.; Lu, Y.; Yu, Q.; Manners, I.; Winnik, M. A., Monitoring Collapse of Uniform Cylindrical Brushes with a Thermoresponsive Corona in Water. ACS Macro Lett. 2018, 7, 166-171.

57. Brewer, A. K.; Striegel, A. M., Characterizing the size, shape, and compactness of a polydisperse prolate ellipsoidal particle via quadruple-detector hydrodynamic chromatography. Analyst 2011, 136, 515-519.

58. De Greef, T. F. A.; Smulders, M. M. J.; Wolffs, M.; Schenning, A. P. H. J.; Sijbesma, R. P.; Meijer, E. W., Supramolecular Polymerization. Chem. Rev. 2009, 109, 5687-5754.

59. Smulders, M. M. J.; Nieuwenhuizen, M. M. L.; de Greef, T. F. A.; van der Schoot, P.; Schenning, A. P. H. J.; Meijer, E. W., How to Distinguish Isodesmic from Cooperative Supramolecular Polymerisation. Chem. Eur. J. 2010, 16, 362-367. 


\section{For Table of Contents Use Only}

“Complementary Nucleobase Interactions Drive the Hierarchical Self-Assembly of Amphiphilic Bottlebrush Block Copolymers Toward Cylindrical Supramolecules”

Spyridon Varlas, Zan Hua, Joseph R. Jones, Marjolaine Thomas, Jeffrey C. Foster,* and Rachel K. O’Reilly*

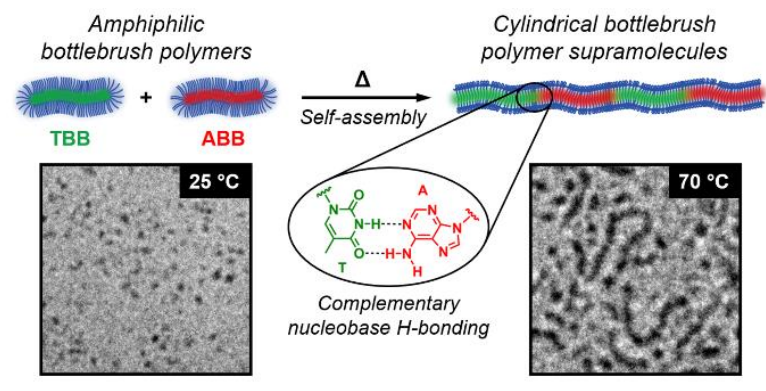

